\title{
Which Costs Matter? Costs Included in Economic Evaluation and their Impact on Decision Uncertainty for Stable Coronary Artery Disease
}

\author{
James Lomas ${ }^{1} \cdot$ Miqdad Asaria $^{1} \cdot$ Laura Bojke $^{1} \cdot$ Chris P. Gale $^{2} \cdot$ Gerry Richardson $^{1} \cdot$ \\ Simon Walker ${ }^{1}$
}

Published online: 14 February 2018

(C) The Author(s) 2018, corrected publication 2019

\begin{abstract}
Background Variation exists in the resource categories included in economic evaluations, and National Institute for Health and Care Excellence (NICE) guidance suggests the inclusion only of costs related to the index condition or intervention. However, there is a growing consensus that all healthcare costs should be included in economic evaluations for Health Technology Assessments (HTAs), particularly those related to extended years of life.

Objective and Methods We aimed to quantify the impact of a range of cost categories on the adoption decision about a hypothetical intervention, and uncertainty around that decision, for stable coronary artery disease (SCAD) based on a dataset comprising 94,966 patients. Three costing scenarios were considered: coronary heart disease (CHD) costs only, cardiovascular disease (CVD) costs and all costs. The first two illustrate different interpretations of what might be regarded as related costs.

Results Employing a 20-year time horizon, the highest mean expected incremental cost was when all costs were included (£2468) and the lowest when CVD costs only were included (£2377). The probability of the treatment being cost effective, estimating health opportunity costs using a ratio of $£ 30,000$ per quality-adjusted life-year
\end{abstract}

Electronic supplementary material The online version of this article (https://doi.org/10.1007/s41669-018-0068-1) contains supplementary material, which is available to authorized users.

James Lomas

james.lomas@york.ac.uk

1 Centre for Health Economics, University of York, York YO10 5DD, UK

2 MRC Bioinformatics Centre, LICAMM, University of Leeds, Leeds, UK
(QALY), was different for each of the CHD (70\%) costs, CVD costs $(73 \%)$ and all costs (56\%) scenarios. The results concern a hypothetical intervention and are illustrative only, as such they cannot necessarily be generalised to all interventions and diseases.

Conclusions Cost categories included in an economic evaluation of SCAD impact on estimates of both cost effectiveness and decision uncertainty. With an aging and co-morbid population, the inclusion of all healthcare costs may have important ramifications for the selection of healthcare provision on economic grounds.

\section{Key Points for Decision Makers}

Variation exists in the resource categories included in economic evaluations, and National Institute for Health and Care Excellence (NICE) guidance suggests the inclusion only of costs related to the index condition or intervention.

Cost categories included in an economic evaluation of stable coronary artery disease significantly impact on estimates of cost effectiveness and decision uncertainty.

\section{Introduction}

Interventions in patients with coronary heart disease (CHD) or at risk of CHD present significant costs to the UK National Health Service (NHS), may reduce the risks of 
CHD events such as acute myocardial infarction (MI), may reduce the risk of other (non-CHD) health events and can potentially improve survival patients $[1,2]$. One example is the prescription of statins (HMG-CoA reductase inhibitors) as primary prevention for $\mathrm{CHD}$, which has attracted controversy due to the potential high up-front cost but a potential overall net saving due to reduced future $\mathrm{CHD}$ costs as well as wider cardiovascular disease (CVD) costs such as those of strokes avoided [1]. Yet the statins may also increase life expectancy and costs will be incurred by the NHS during these extended years of life [3, 4].

In an economic evaluation the incremental costs incurred by a new intervention and the health benefit it generates are compared to the health benefit of activities that could have been funded with the same resources elsewhere [5]. There is extensive debate, however, around what types of costs should be included in estimating the incremental costs of a new intervention [6, 7], and what costs are included can have material impacts on the expected cost effectiveness and the associated uncertainty. This can affect the decisions reached by policy makers, e.g. whether to approve or reject the new intervention unconditionally, or whether to recommend an alternative coverage decision such as coverage with evidence development [8-10]. This paper considers the importance of the choice of costs to include using a hypothetical intervention that reduces the risk of CVD events in patients with stable coronary artery disease (SCAD).

The debate considers two types of costs: related and unrelated. Garber and Phelps [11] define unrelated costs as those that are independent of the intervention under consideration, and in cases where treatments extend life costs are defined as unrelated if they are independent of treatment but conditional on survival. Conversely, all other costs that are not independent are defined as related. National Institute for Health and Care Excellence (NICE) guidance [12] explicitly makes the recommendation that "costs that are considered to be unrelated to the condition or technology of interest should be excluded". 1 A posited theoretical justification for this recommendation is that the aim of economic evaluation is to judge each intervention on its own 'merits' [13]. For example, it is argued that the inclusion of future unrelated costs can increase the likelihood of a life-extending technology being judged to not be cost effective even at zero price, which may seem counterintuitive [7, 14]. The second US Panel on Cost-effectiveness in Health and Medicine, however, recommends that all current and future, related and unrelated healthcare costs should be included in cost-effectiveness analysis.

\footnotetext{
1 A fuller discussion of guidelines and recommendations pertaining to the exclusion of unrelated costs (in particular those in extended years of life) can be found elsewhere [41].
}

This reflects a growing consensus that all healthcare costs should be included, particularly in extended years of life $[3,15]$. Indeed, the guidelines employed in the Netherlands and by the LFN (Dental and Pharmaceutical Benefits Board) in Sweden [16] also recommend the inclusion of future unrelated costs [17]. ${ }^{2}$

In practice, whether costs are related or unrelated is based on a judgement of whether they are plausibly related to either the condition or the intervention being considered. This is a subjective assessment and is argued to result in analyses that impose arbitrary restrictions on the costs that are considered relevant [18]. Further, it is not always clear which costs are related and which are unrelated to the condition or intervention. One such example is the case of acute MI and, in particular, how it affects survivors in terms of life expectancy and the likelihood of experiencing health events or diseases in later life $(20.72 \%$ of patients with SCAD are MI survivors in the CALIBER [ClinicAl research using Linked Bespoke studies and Electronic Records] dataset [19]). MI survivorship is increasing due to the availability of effective treatments; for example, in England in 2010, on average, $27.32 \%$ of MI cases that were admitted to hospital died within 30 days, where the corresponding 2002 proportion was $37.18 \%$ [20].

A review of published economic evaluations of the treatment of MI finds considerable variation in the types of costs included, reflecting both the subjectivity in determining relevance and variation in recommendations among guidelines. Nineteen economic evaluations published since 2006 are categorised according to the cost categories included: CHD costs only, CVD costs only or all costs (see Electronic Supplementary Material Appendix). CHD costs form the smallest cost category, including costs exclusively related to $\mathrm{CHD}$, e.g. costs attributable to MI. CVD costs include not only all costs attributable to CHD but all costs related to the cardiovascular system more broadly, e.g. ischaemic and haemorrhagic stroke. The CHD costs category is therefore a subset of the CVD costs category. These categories are both subsets of the all costs category, where all costs are included regardless of to which health conditions they are attributable, e.g. they therefore include CVD and non-CVD costs such as those attributable to cancer. Of the 19 studies, five were found to include CHD costs only, eight CVD costs only and six evaluations included all costs. The main difference between studies including CVD costs rather than CHD costs was that costs from stroke events were included. Studies considering related costs only tended to be those that were trial-based analyses, where only CHD/CVD-related data were recorded, or

\footnotetext{
2 A further debate surrounds the inclusion of non-healthcare costs, as recommended by the guidelines of the Netherlands and Sweden. This remains more controversial and is beyond the scope of this paper.
} 
model-based approaches including only CHD or CVD events. One observational data study considered only CVD costs where the analysis was performed on a dataset with detailed information collected on only a select number of CVD endpoints [21]. Of those studies that incorporated non-CVD costs, it is informative to consider the different approaches taken. One study was based on a trial that had collected a wide range of types of resource use [22]. ${ }^{3}$ Model-based analyses used a number of different approaches: national averages [23], age-specific costs from riskadjustment studies [24, 25], estimates of lifetime costs obtained from registry data and use of expert opinion [26]. Finally, for one study undertaking an analysis of observational data, all costs were included in the analysis as the default, but a sensitivity analysis was undertaken in which only costs with the ischaemic heart diseases International Classification of Diseases (ICD) code were included [27].

Given the variation in practice by analysts in conducting economic evaluations of MI treatment and the difficulty in distinguishing what costs are related from those that are not, it is important to consider the implications of which costs are included in an economic evaluation. In this paper a recently published 'real-world' cost-effectiveness model is used to consider a hypothetical intervention that reduces the risk of CVD health events for patients with SCAD to investigate the impact of including different cost categories on expected cost effectiveness and decision uncertainty. This analysis represents the first attempt to consider the implications of collecting broader cost data not only in terms of addressing concerns around the expected incremental costs, but also in terms of any impacts upon the associated decision uncertainty.

\section{Methods}

In order to illustrate and quantify the importance, in terms of incremental cost and decision uncertainty, of the analyst's decision around which cost categories to include, three different scenarios are specified using the CALIBER model [28]. The three scenarios pertain to the range of costs that are included in each analysis: CHD costs only, CVD costs or all costs. Full details of the model are presented elsewhere [28] but, in brief, the CALIBER model employs a set of risk equations estimating probabilities of subsequent MI, ischaemic and haemorrhagic stroke and mortality that are structured as a Markov model to model disease progression in a secondary prevention of $\mathrm{CHD}$

\footnotetext{
3 Interestingly, in this study one of the resource use measures that was particularly high for one patient among those treated was considered by the authors to only be "weakly related" to the intervention and so was excluded as part of the sensitivity analysis, which had quite a large effect on the mean cost difference [22].
}

context [28]. These equations are estimated using data from four data sources (detailed in Denaxas et al. [29]): the Clinical Practice Research Database (CPRD), Myocardial Ischaemia National Audit Project (MINAP) registry, Hospital Episode Statistics (HES) and Office for National Statistics (ONS). ${ }^{4}$ It is assumed that a hypothetical treatment administered to patients in the SCAD health state at a cost of $£ 250$ per year reduces the probability of potentially fatal cardiovascular health events compared with a standard care control group, with a relative risk of 0.8. The patient cohort modelled is assumed to be in the fifth decile of 5-year risk of a composite CVD first event.

The model is evaluated probabilistically, with 999 iterations run in total. For each iteration of the model eight key results are recorded: quality-adjusted life-years (QALYs) associated with the intervention and control groups and costs associated with the intervention and control groups, according to three different costing scenarios. Two of the three scenarios represent plausible approaches to including only related costs as per the NICE guidance on this issue, one of which is narrow and the other slightly broader. In the CHD costs only and CVD costs only scenarios, only healthcare costs that are identifiable as CHD and CVD through the ICD 10th edition (ICD-10) codes of the recorded Health Resource Group (HRG) are included. ${ }^{5}$ The third scenario includes all healthcare costs and so represents an approach where no distinction is drawn in terms of related and unrelated costs. In all scenarios, incremental discounted costs and QALYs are evaluated at a time horizon of 20 years (equivalent to a lifetime time horizon given the starting age of the cohort [mean age at cohort entry was 65 years for males and 73 years for females], where the lifetime time horizon is the recommended choice for treatments that will impact on mortality [5]) using an annual discount rate of 3.5\% for both costs and QALYs, and the net health benefit is calculated using $£ 30,000$ per QALY [12]. It is not possible within this model to separate out the implications of including different cost categories into effects from unrelated costs in extended years of life and those in years of life not extended by the intervention as the treatment has both morbidity and mortality impacts. However, the relative size of the effect of extended years of life will be smaller with shorter time horizons and so we present some results to show the effect of time horizon on cost effectiveness and decision uncertainty.

\footnotetext{
${ }^{4}$ Further details given in Asaria et al. [28] and Walker et al. [42]. Resource use is taken from administrative data sources, CPRD and HES, and then costed on the basis of NHS reference costs, Personal Social Services Research Unit (PSSRU) unit costs and NHS prescription cost analysis [43-45]. Estimation of cost parameters in the model is undertaken using the panel data approach outlined in Walker et al. [42].

${ }^{5}$ CHD HRGs were defined as those with ICD-10 codes from I10 through to I52.
} 


\section{Results}

Cost results are first considered in isolation. The mean costs in the treated and control groups are presented along with summary statistics of the incremental costs, illustrating the effect of the three different scenarios on the location and dispersion of the distribution of estimated incremental costs, in Table 1.

Mean costs over a 20-year time horizon for both the treated and control groups increase with the inclusion of additional cost categories. In terms of incremental costs, including all costs results in the highest mean incremental cost. The CVD costs only scenario gives the lowest mean incremental cost. Variability of incremental costs increases when more cost categories are included based on the width of the $95 \%$ credibility interval. The distribution of incremental costs is presented in Fig. 1.

Figure 1 shows the distributions of incremental costs under each scenario. Mean incremental costs were lowest in the CVD costs only scenario and highest in the all costs scenario. The distributions of incremental costs under scenarios with more cost categories included are considerably wider, reflecting greater variability.

To investigate the relative effect of unrelated costs in extended years of life compared with those in life-years not extended by the intervention, we investigate the distribution of incremental costs for different time horizons up to 20 years, with changes over time likely to reflect the increasing impact of costs in extended years of life. These results are summarised in Figs. 2 and 3.

Table 1 Summary statistics of costs and incremental costs at a time horizon of 20 years

\begin{tabular}{lrrr}
\hline Statistics & \multicolumn{3}{c}{ Resource categories included } \\
\cline { 2 - 4 } & CHD (£) & CVD (£) & All (£) \\
\hline Mean $c_{\mathrm{t}}$ & 29,117 & 36,310 & 50,202 \\
Mean $c_{\mathrm{c}}$ & 26,721 & 33,934 & 47,734 \\
Mean $c_{\mathrm{t}}-c_{\mathrm{c}}$ & 2396 & 2377 & 2468 \\
Minimum $c_{\mathrm{t}}-c_{\mathrm{c}}$ & 2287 & 2232 & 2273 \\
Maximum $c_{\mathrm{t}}-c_{\mathrm{c}}$ & 2523 & 2547 & 2686 \\
Range $c_{\mathrm{t}}-c_{\mathrm{c}}$ & 237 & 315 & 414 \\
Standard deviation $c_{\mathrm{t}}-c_{\mathrm{c}}$ & 39 & 53 & 69 \\
Median $c_{\mathrm{t}}-c_{\mathrm{c}}$ & 2394 & 2374 & 2468 \\
Q $0.025 c_{\mathrm{t}}-c_{\mathrm{c}}$ & 2323 & 2281 & 2343 \\
Q $0.975 c_{\mathrm{t}}-c_{\mathrm{c}}$ & 2471 & 2478 & 2595 \\
$95 \%$ credibility interval $c_{\mathrm{t}}-c_{\mathrm{c}}$ & 148 & 197 & 252 \\
\hline
\end{tabular}

CHD coronary artery disease, $C V D$ cardiovascular disease, $c_{c}$ cost when control, $c_{t}$ cost when treated, $Q_{0.025} 2.5$ th percentile, $Q_{0.975}$ 97.5th percentile
Figure 2 shows the credibility interval of the incremental costs for two different cost category scenarios (CHD and all costs) against the time horizon. It can be seen that with longer time horizons, such as 20 years, the all costs scenario produces higher incremental costs on average. Looking at the shorter time horizons the opposite is found, with incremental costs on average higher under the CHD scenario. Variability, seen from the width of the credibility interval shown in Fig. 3, is higher for the all costs scenario at all time horizons. For both scenarios, variability increases with the time horizon.

It is also useful to consider the impact of incremental costs on cost effectiveness in order to illustrate how these findings could affect decision-making and decision uncertainty. In all scenarios the mean incremental QALY from treatment is 0.084 . These are the denominators in the estimates of the incremental cost-effectiveness ratios (ICERs) provided in Table 2, where the numerators are the mean incremental costs reported in Table 1.

For illustrative purposes we consider a decision maker who regards a treatment as cost effective when the ICER is less than $£ 30,000$ per QALY gained [12]. Whilst the treatment is expected to be cost effective in all scenarios (all of the ICERs are below $£ 30,000$ per QALY), it can be seen that the choice of cost categories that are included is likely to influence the degree of decision uncertainty. Figure 4 shows the distributions of net health benefits for the treatment. The treatment is cost effective when the net health benefit is greater than zero.

The proportion of iterations with a positive net health benefit, i.e. the probability of being cost effective, varies across the three scenarios: CHD costs (70\%), CVD costs (73\%) and all costs (56\%). These percentages reflect the distribution of net health benefit in the different scenarios where both the location and dispersion of the distributions is influenced by the choice of cost categories to be included.

\section{Discussion}

This paper assesses the cost effectiveness of a hypothetical intervention using a previously published model based on 'real-world' data to demonstrate that the inclusion of unrelated costs will affect both the mean incremental costs, ICER and decision uncertainty.

Unambiguously, costs will be greater when more categories are included (given costs are non-negative). It is harder to predict how the different cost category scenarios will affect the estimated incremental costs that are required for economic evaluation. The treatment under consideration here reduces the risk of cardiovascular events, each with associated CHD, CVD and non-CVD costs, in normal 


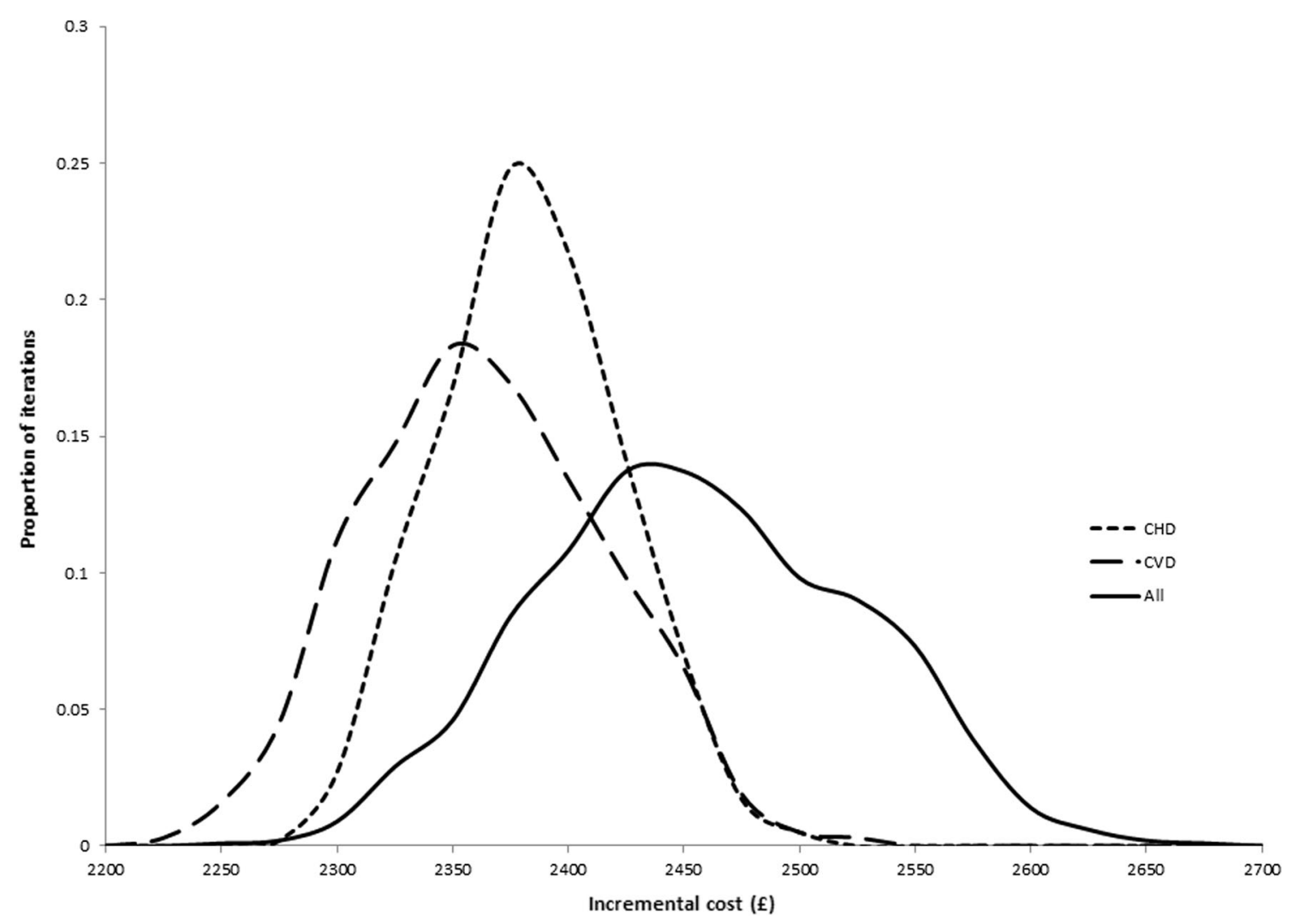

Fig. 1 Frequency plot of incremental costs under three scenarios. CHD coronary heart disease, CVD cardiovascular disease

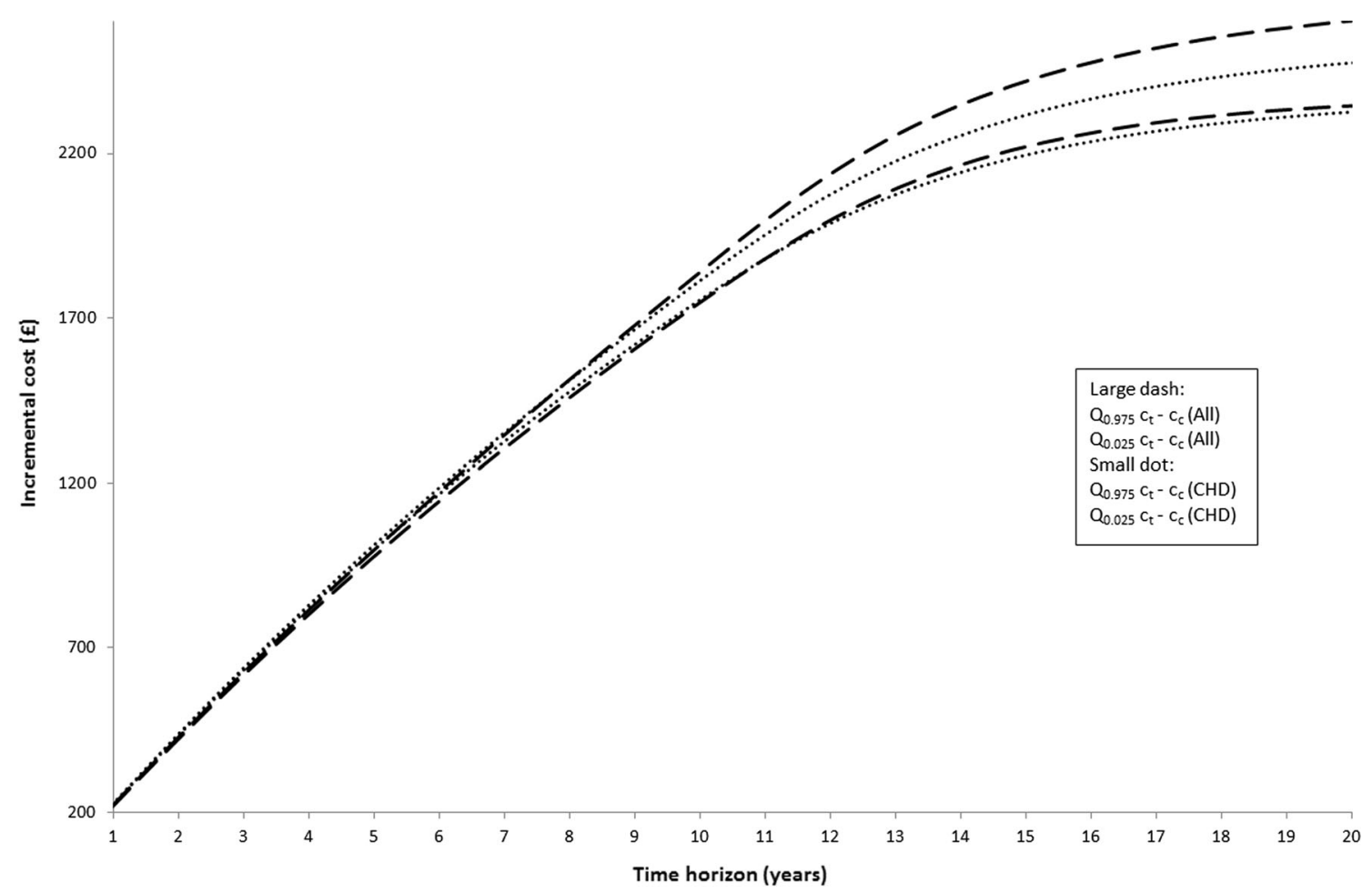

Fig. 2 95\% credibility interval (CI) against time horizon by costing scenario. CHD coronary artery disease, $c_{c}$ cost when control, $c_{t}$ cost when treated, $Q_{0.025} 2.5$ th percentile, $Q_{0.975} 97.5$ th percentile 


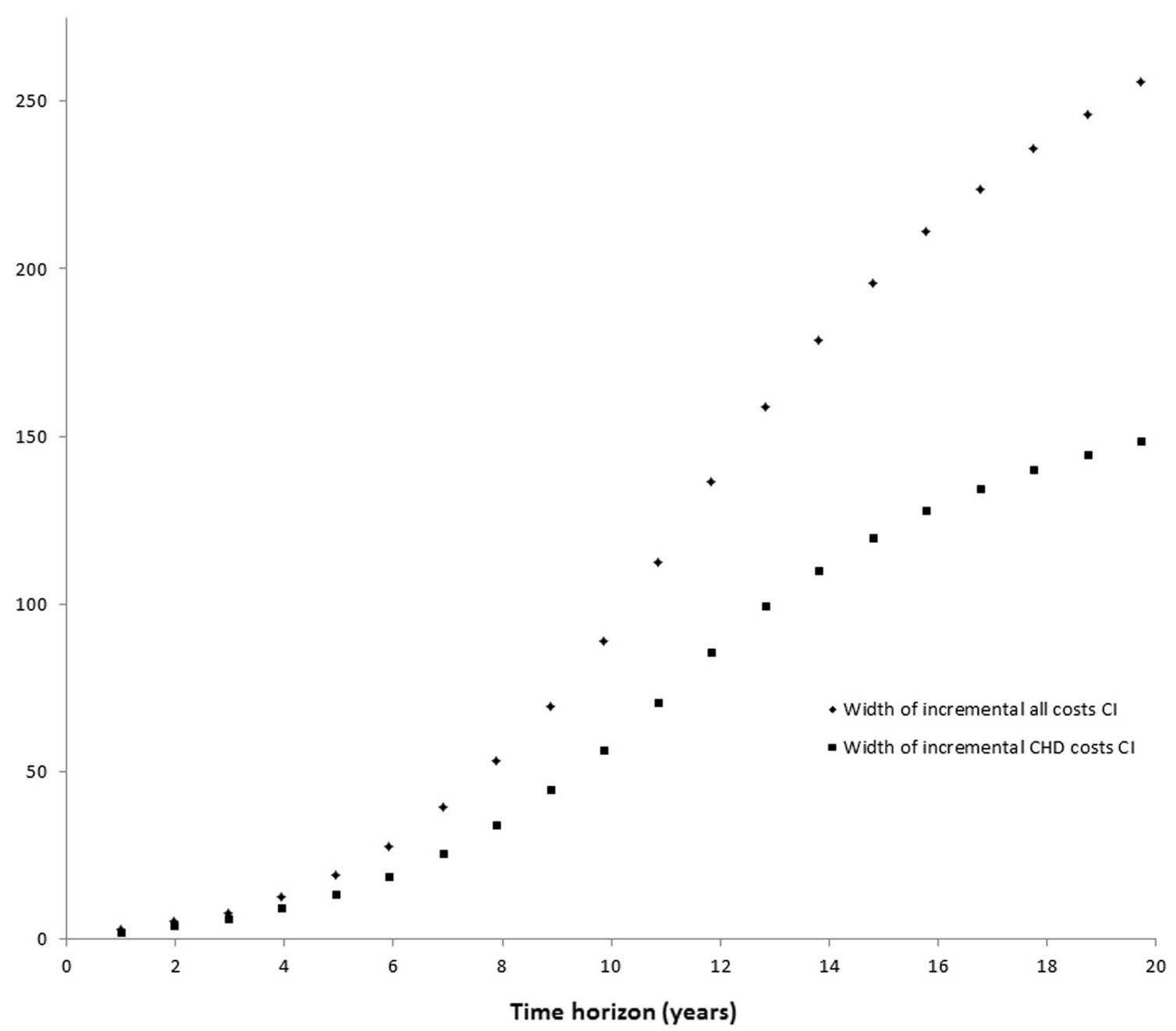

Fig. 3 Width of $95 \%$ credibility interval against time horizon by costing scenario. $C H D$ coronary heart disease, $C I$ 95\% credibility interval

Table 2 Estimated incremental cost-effectiveness ratios in the different costing scenarios

\begin{tabular}{llll}
\hline & \multicolumn{2}{l}{ Resource categories included } & All \\
\cline { 2 - 4 } & CHD & CVD & $£ 29,485$ per QALY
\end{tabular}

CHD coronary heart disease, $C V D$ cardiovascular disease, ICER incremental cost-effectiveness ratio, $Q A L Y$ quality-adjusted life-year years of life (i.e. not extended years of life). Each of these events is potentially fatal, and so the treatment extends life. This distinction is important when thinking about the overall effect on incremental costs. In normal years of life, including more cost categories increases the magnitude of the cost savings that are brought about by the reduced risk of cardiovascular events. ${ }^{6}$ However, including additional cost categories means greater costs in extended years of life resulting from the treatment. As such, these two effects offset one

\footnotetext{
${ }^{6}$ Note that this holds only if the excluded cost categories are not truly unrelated.
}

another to some extent. ${ }^{7}$ Therefore, the overall effect is ambiguous. Moving from only CHD costs to CVD costs reduces the mean incremental cost, because more cost savings (e.g. from reduced strokes) are captured during normal years of life than the additional incremental CVD costs captured in extended years of life resulting from the treatment. However, moving from either CHD costs only or CVD costs only to including all costs increases the incremental costs overall because the extended years of life effect outweighs the effect of capturing more savings during normal years of life.

$\overline{7}$ Authors have noted this kind of offset in previous applications [46]. 


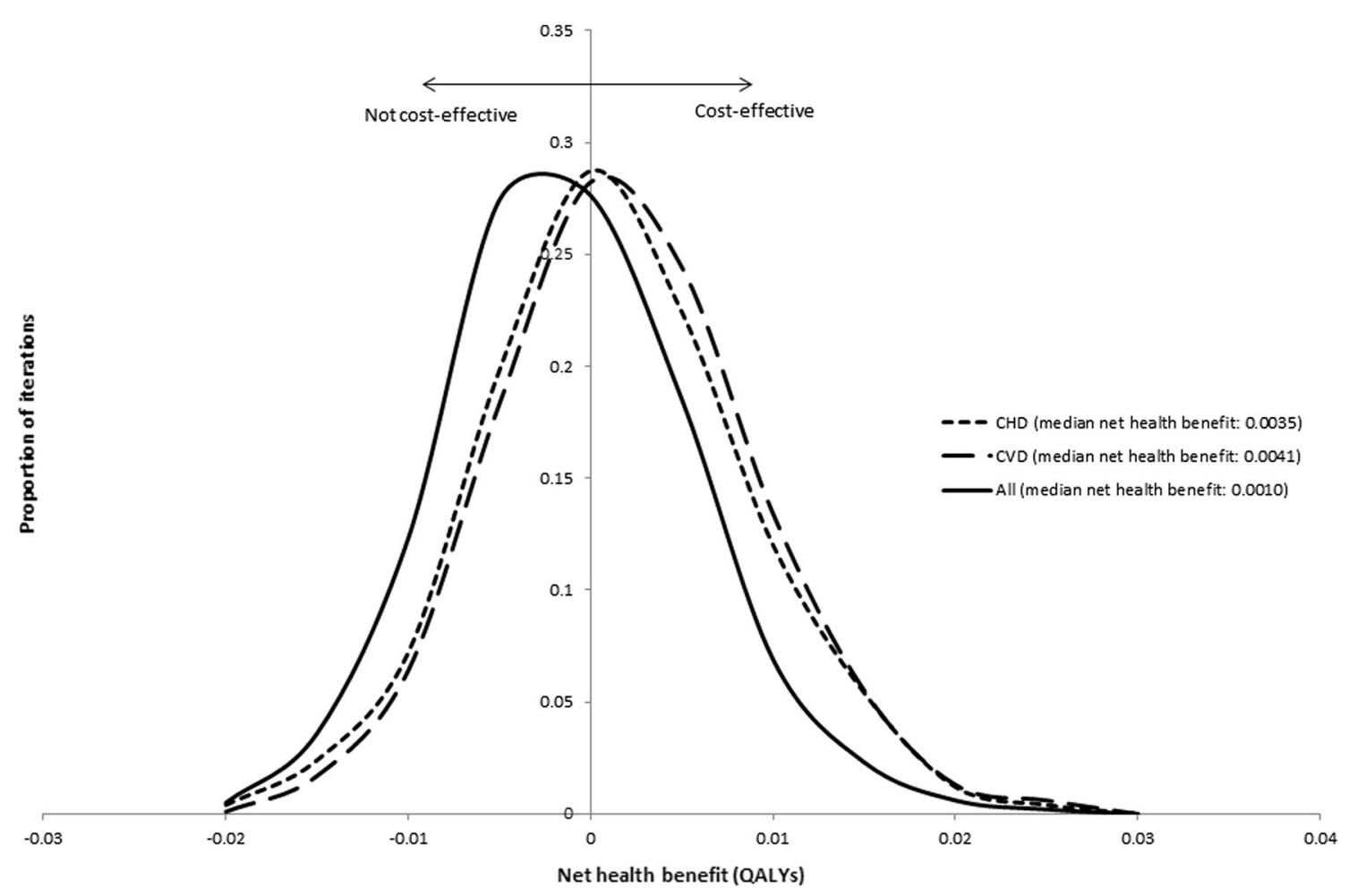

Fig. 4 Frequency plot of net health benefits under three scenarios. $C H D$ coronary heart disease, $C V D$ cardiovascular disease, $Q A L Y s$ qualityadjusted life-years

Unfortunately, given the model used, it is not possible to separate out these effects and only the joint effect is observable. However, by considering shorter time horizons we can examine the relative impacts of costs in normal and extended years of life. With a shorter time horizon the effect of costs in normal years of life is relatively more influential and so the direction of effect on the cost effectiveness of the ambiguous relationship between inclusion of broader cost categories may vary with the time horizon. Indeed, this is what is seen in our results. At very short time horizons, the incremental costs are on average higher when restricted to CHD costs only than when including all cost categories. The variability of costs is higher for all costs than it is for CHD costs at all time horizons analysed. This would translate to greater decision uncertainty when including all costs, ceteris paribus, even when using short time horizons.

In addition to the effect on mean incremental costs, this paper also explores the effect that the different scenarios have on decision uncertainty. Again, this effect needs to be thought of as two separate underlying processes. The first of these is, when considering a technology that has positive incremental costs and incremental QALYs, the proximity of the mean ICER to the 'cost-effectiveness threshold' being used in the analysis. ${ }^{8}$ When the ICER is close to the

\footnotetext{
${ }^{8}$ In other words, the proximity of the mean net health benefit of the technology to zero.
}

'cost-effectiveness threshold', ceteris paribus, then it is more likely that the decision is going to be uncertain. The other factor is the uncertainty of the estimated ICER (in this case driven by the variability in incremental costs). The variability of costs (and incremental costs) is likely to increase with the addition of cost categories, unless a negative correlation exists between the costs within the narrower category and those only within the broader category.

Where there is no extension of life effect from a treatment, including unrelated costs will not affect the mean ICER, in expectation, as these additional costs would be the same on average regardless of treatment received. Decision uncertainty, however, will be affected given the variability of costs. As such, under these circumstances, the addition of truly unrelated costs amounts to adding noise and increased variability into the model.

Adding noise and variability can lead to greater uncertainty and there are two reasons why this might be a concern. The first is that there will be reduced power to detect a statistically significant effect, since standard errors will increase. The second concern, more prevalent within health economic evaluation, is that the decision uncertainty will be inflated, thus reducing the probability of making the right decision. Of course, the analyst will never know what is truly related and truly unrelated and so identifying something as noise with certainty is impossible. This is not 
to say that unrelated costs should never be included because of the potential of introducing noise, but it is important to consider the implications of adding in what might be truly unrelated costs in normal years of life. This issue has been overlooked in the literature on this topic to date.

As already discussed, there is reason to caution against including unrelated costs in some cases where there is no (or little) mortality effect. In addition, obtaining data on unrelated costs may not be without cost to the analyst, especially obtaining data that are both very unlikely to be related and are realised many years in the future-although this may be getting less costly with time [4] as a growing number of sources exist that can be used to provide data for unrelated costs in extended years of life across a number of different countries [30-34].

Lack of appropriate and robust data will be an issue in many circumstances. Experimental evidence typically does not have a sufficiently long time horizon to identify the occurrence of all future related and unrelated events and their associated costs. As an alternative, the use of observational data from administrative sources is becoming increasingly prevalent and is well-suited to estimate parameters with large numbers of observations. Observational data are typically collected over a longer period of time; however, by design it is not as robust as randomised trial data when it comes to estimating the incremental cost caused by treatment, with potential bias coming from unobserved confounding and selection effects. With both randomised controlled trial (RCT) and observational evidence there is the issue of sufficient breadth of study design to identify all related and unrelated costs. By their very nature, such studies would be unlikely to be focused on collecting events unrelated to the primary intervention and, as such, data collection would have to be sufficiently flexible or broad to capture all costs (related and unrelated). Routine data sources, such as HES in the UK context, which capture all healthcare-related interventions and hospital visits are another possibility. HES has recently been used to compute average costs by age, sex and index of multiple deprivation (IMD) group [34]. This source has the potential to be very useful for incorporation into decision models, but there are two limitations that provide scope for future research. The first is that the costs in this paper include both related and unrelated costs, meaning that researchers would need to adapt its results in order to include it alongside related costs in extended years of life estimated as part of the model [33]. The second limitation is that the costs of the patient population at hand may not be well represented by the national average on account of the relationship between costs and co-morbidities. In such circumstances, disentangling the associations between costs, age and morbidities will be required for the precise estimation of parameters capturing unrelated costs. There is already a growing understanding of the causal effect that age has on costs, which has been found to be questionable. In the first instance, age was considered to be a 'red herring', with costs increasing with age due only to a spurious association, and the underlying effect on costs deriving from time until death and not age itself [35, 36]. More recently, research has started to question whether this red herring hypothesis is itself a red herring, with researchers analysing the effects of morbidity and multi-morbidity on costs in greater depth [37, 38]. Despite these limitations and challenges it is likely that, with further research, electronic health records will be increasingly utilised for the incorporation of unrelated costs in extended years of life where this is deemed appropriate.

Even in the absence of observational data, it is still possible to place a reasonable estimate on these future unrelated costs parameters. There are methods that can be used to illustrate the potential range values for these parameters, reflecting their degree of uncertainty [39]. This can then be used to guide future research priorities [40]. To inform these uncertain estimates, expert elicitation methods could be useful. In some circumstances, even where long-term observational data are not available, clinical experts may have experience of observing events occurring in survivors of acute MI over a long enough period. This clinical experience can be used to generate distributions for these unrelated future costs, reflecting a large amount of uncertainty around the estimates. All things considered, lack of data is certainly a challenge, but this seems more of a practical obstacle to overcome (if these data are potentially influential to the decision) rather than a reason for exclusion.

\subsection{Limitations}

Because the model itself concerns a hypothetical intervention, the specific results should not be over-interpreted, nor can they be generalised for assessments of how much of an impact the inclusion of unrelated costs will have in different contexts. The results do allow us to identify the types of effects that will result from including unrelated costs. To put this another way, this paper contributes to the literature by illustrating which costs matter and why, but should not be used to say how much different costs matter outside of the context analysed. For example, within the context analysed, it can be seen that the variability of incremental costs is generally low, irrespective of which costing scenario is used. This results from the large number of observations upon which the system of equations is estimated, and hence granting a degree of precision that would not be attainable in smaller studies. 


\section{Conclusions}

It is suggested that economic evaluations should include estimates of incremental healthcare costs that fall within the healthcare budget and not just the intervention costs associated with the treatment. However, in moving beyond intervention costs, the analyst faces challenges in determining which healthcare costs (unrelated and unrelated) are truly caused by the treatment, which can be particularly demanding when there are variable and noisy cost data, and when the knowledge around the natural history of a disease is not well-known, as in the case of acute MI survivorship. With an aging and co-morbid population, the inclusion of all healthcare costs may have important ramifications for the selection of healthcare provision on economic grounds.

This paper contributes to this discussion by illustrating the importance of different types of costs-which costs matter-in the context of SCAD, where little is known about what is truly related or unrelated and there is considerable variation in analytical practice concerning cost categories to include in economic evaluation. It has been shown that costs matter, not just in terms of the point estimate of cost effectiveness, the expected ICER, but also in terms of decision uncertainty. Where adding unrelated costs in evaluations without an impact on survival, this may simply add noise to the data and therefore will reduce the probability of making the right decision.

The inclusion of unrelated costs is important, especially when the treatment is expected to extend life, and so including all cost categories is at least recommended as a sensitivity analysis (if the associated cost to analyst of obtaining data is not prohibitively high).

Acknowledgements We are grateful to Pieter van Baal for comments on an earlier draft of this paper. Participants at the University of York Health Sciences Seminar, and in particular Karen Bloor, are thanked for their observations and suggestions. Finally, this paper was the basis of a basis of a poster presentation at the 2016 ISPOR (International Society for Pharmacoeconomics and Outcomes Research) meeting in Vienna, Austria; we would like to thank the participants, whose feedback was greatly appreciated.

Author Contributions James Lomas led the drafting of the manuscript and produced the summaries of results of the analysis, as well as contributing to the production and interpretation of the results. Miqdad Asaria provided comments on several draft versions of the manuscript, contributed to the conceptualisation of the analysis and produced the code for the analysis undertaken. Laura Bojke contributed to the initial concept of the paper and drafted parts of the manuscript, provided extensive comments on previous draft versions and contributed to the interpretation of the results. Chris P. Gale contributed to the initial concept of the paper and provided comments on previous draft versions of the manuscript. Gerry Richardson contributed to the initial concept of the paper and drafted parts of the manuscript, provided extensive comments on previous draft versions and contributed to the interpretation of the results. Simon Walker drafted parts of the manuscript, provided extensive comments on several draft versions of the manuscript, contributed to the conceptualisation of the analysis as well as contributing to the production and interpretation of the results.

\section{Compliance with Ethical Standards}

Funding The research was funded by the National Institute for Health Research (NIHR) Collaboration for Leadership in Applied Health Research and Care, Yorkshire and Humber (www.clahrc-yh. nihr.ac.uk). The views expressed are those of the author(s), and not necessarily those of the NHS, the NIHR or the Department of Health.

Conflict of interest No conflicts of interest exist for James Lomas, Miqdad Asaria, Laura Bojke, Chris P. Gale, Gerry Richardson and Simon Walker.

Transparency declaration The lead author affirms that this manuscript is an honest, accurate and transparent account of the study being reported; that no important aspects of the study have been omitted; and that any discrepancies from the study as planned (and, if relevant, registered) have been explained.

Data sharing statement No additional data are available.

Patient involvement statement Not applicable.

Patient consent statement Not applicable.

Open Access This article is distributed under the terms of the Creative Commons Attribution 4.0 International License (http:// creativecommons.org/licenses/by/4.0/), which permits unrestricted use, distribution, and reproduction in any medium, provided you give appropriate credit to the original author(s) and the source, provide a link to the Creative Commons license, and indicate if changes were made.

\section{References}

1. Hawkes N. NICE guidelines could put 12 million UK adults on statins. BMJ. 2017;358:j3674.

2. NICE. Transcatheter aortic valve implantation for aortic stenosis. NICE; 2017. https://www.nice.org.uk/guidance/ipg586/chapter/1Recommendations. Accessed 4 Aug 2017.

3. Morton A, Adler AI, Bell D, Briggs A, Brouwer W, Claxton K, et al. Unrelated future costs and unrelated future benefits: reflections on NICE guide to the methods of technology appraisal. Health Econ. 2016;25(8):933-8.

4. van Baal P, Meltzer D, Brouwer W. Future costs, fixed healthcare budgets, and the decision rules of cost-effectiveness analysis. Health Econ. 2016;25(2):237-48.

5. Drummond M, Sculpher M, Claxton K, Stoddart G, Torrance G. Methods for the economic evaluation of health care programmes. 4th ed. Oxford: Oxford University Press; 2015.

6. Meltzer D. Future costs in medical cost-effectiveness analysis. In: The Elgar companion to health economics. 2nd ed. Edward Elgar Publishing; 2012.

7. van Baal P, Morton A, Brouwer W, Meltzer D, Davis S. Should cost effectiveness analyses for NICE always consider future unrelated medical costs? BMJ. 2017;10(359):j5096.

8. Walker S, Sculpher M, Claxton K, Palmer S. Coverage with evidence development, only in research, risk sharing, or patient 
access scheme? A framework for coverage decisions. Value Health. 2012;15(3):570-9.

9. Claxton K, Palmer S, Longworth L, Bojke L, Griffin S, McKenna $\mathrm{C}$, et al. Informing a decision framework for when NICE should recommend the use of health technologies only in the context of an appropriately designed programme of evidence development. Health Technol Assess. 2012;16(46):1-323.

10. Claxton K, Palmer S, Longworth L, Bojke L, Griffin S, Soares M, et al. A comprehensive algorithm for approval of health technologies with, without, or only in research: the key principles for informing coverage decisions. Value Health. 2016;19(6):885-91.

11. Garber AM, Phelps CE. Future costs and the future of cost-effectiveness analysis. J Health Econ. 2008;27(4):819-21.

12. NICE. Guide to the methods of technology appraisal. NICE; 2013. https://www.nice.org.uk/process/pmg9/chapter/foreword. Accessed 31 Jul 2017.

13. Russell LB. Is prevention better than cure? Brookings Institution; 1986. 134 p. https://books.google.co.uk/books/about/Is Prevention_Better_Than_Cure.html?id=9S5bDPZy6j0C\&redir_ esc=y. Accessed 16 Nov 2017.

14. Davis S, Akehurst R. How do we evaluate technologies that are not cost effective at zero price? Value and Outcomes Spotlight. 2016;359:6-8. https://www.ispor.org/health-policy_ technologies_costs.pdf. Accessed 16 Nov 2017.

15. Sanders GD, Neumann PJ, Basu A, Brock DW, Feeny D, Krahn $\mathrm{M}$, et al. Recommendations for conduct, methodological practices, and reporting of cost-effectiveness analyses. JAMA. 2016;316(10):1093.

16. LFN. General guidelines for economic evaluations from the Pharmaceutical Benefits Board. 2003. https://www.tlv.se/Upload/ English/Guidelines-for-economic-evaluations-LFNAR-2003-2. pdf. Accessed 31 Jul 2017.

17. Zorginstituut Nederland. Richtlijn voor het uitvoeren van economische evaluaties in de gezondheidszorg. 2015. https:// www.zorginstituutnederland.nl/publicaties/publicatie/2016/02/ 29/richtlijn-voor-het-uitvoeren-van-economische-evaluaties-inde-gezondheidszorg. Accessed 16 Nov 2017.

18. van Baal PHM, Feenstra TL, Hoogenveen RT, Ardine de Wit G. Brouwer WBF. Unrelated medical care in life years gained and the cost utility of primary prevention: in search of a "perfect" cost-utility ratio. Health Econ. 2007;16(4):421-33.

19. Rapsomaniki E, Shah A, Perel P, Denaxas S, George J, Nicholas $\mathrm{O}$, et al. Prognostic models for stable coronary artery disease based on electronic health record cohort of 102,023 patients. Eur Heart J. 2014;35(13):844-52.

20. Townsend N, Wickramasinghe K, Bhatnagar P, Smolina K, Nichols M, Leal J, et al. Coronary heart disease statistics: a compendium of health statistics. 2012th ed. London: British Heart Foundation; 2012.

21. Wailoo A, Goodacre S, Sampson F, Alava MH, Asseburg C, Palmer S, et al. Primary angioplasty versus thrombolysis for acute ST-elevation myocardial infarction: an economic analysis of the National Infarct Angioplasty project. Heart. 2010;96(9):668-72.

22. Fitzgerald P, Goodacre SW, Cross E, Dixon S. Cost-effectiveness of point-of-care biomarker assessment for suspected myocardial infarction: the Randomized Assessment of Treatment Using Panel Assay of Cardiac Markers (RATPAC) Trial. Acad Emerg Med. 2011;18(5):488-95.

23. Welsh RC, Sauriol L, Zhang Z, Kolm P, Weintraub WS, Theroux P. Cost-effectiveness of enoxaparin compared with unfractionated heparin in ST elevation myocardial infarction patients undergoing pharmacological reperfusion: a Canadian analysis of the Enoxaparin and Thrombolysis Reperfusion for Acute Myocardial Infarction Treatment-Thrombolysis in Myocardial Infarction (ExTRACT-TIMI) 25 trial. Can J Cardiol. 2009;25(12):e399-405.
24. Choudhry NK, Patrick AR, Antman EM, Avorn J, Shrank WH. Cost-effectiveness of providing full drug coverage to increase medication adherence in post-myocardial infarction medicare beneficiaries. Circulation. 2008;117(10):1261-8.

25. Ladapo JA, Jaffer FA, Weinstein MC, Froelicher ES. Projected cost-effectiveness of smoking cessation interventions in patients hospitalized with myocardial infarction. Arch Intern Med. 2011;171(1):39-45.

26. Berg J, Lindgren P, Spiesser J, Parry D, Jönsson B. Cost-effectiveness of clopidogrel in myocardial infarction with ST-segment elevation: a European model based on the CLARITY and COMMIT trials. Clin Ther. 2007;29(6):1184-202.

27. Bäumler M, Stargardt T, Schreyögg J, Busse R. Cost effectiveness of drug-eluting stents in acute myocardial infarction patients in Germany. Appl Health Econ Health Policy. 2012;10(4):235-48.

28. Asaria M, Walker S, Palmer S, Gale CP, Shah AD, Abrams KR, et al. Using electronic health records to predict costs and outcomes in stable coronary artery disease. Heart. 2016;102(10):755-62.

29. Denaxas SC, George J, Herrett E, Shah AD, Kalra D, Hingorani $\mathrm{AD}$, et al. Data resource profile: cardiovascular disease research using linked bespoke studies and electronic health records (CALIBER). Int J Epidemiol. 2012;41(6):1625-38.

30. Meltzer D, Egleston B, Stoffel D, Dasbach E. Effect of future costs on cost-effectiveness of medical interventions among young adults. Med Care. 2000;38(6):679-85.

31. Manns B, Meltzer D, Taub K, Donaldson C. Illustrating the impact of including future costs in economic evaluations: an application to end-stage renal disease care. Health Econ. 2003;12(11):949-58.

32. Kruse M, Sørensen J, Gyrd-Hansen D. Future costs in cost-effectiveness analysis: an empirical assessment. Eur J Health Econ. 2012;13(1):63-70.

33. van Baal PHM, Wong A, Slobbe LCJ, Polder JJ, Brouwer WBF, de Wit GA. Standardizing the inclusion of indirect medical costs in economic evaluations. Pharmacoeconomics. 2011;29(3):175-87.

34. Asaria M. Health care costs in the English NHS: reference tables for average annual NHS spend by age, sex and deprivation group. CHE Research Paper, no. 147. York: Centre for Health Economics, University of York; 2017. https://pure.york.ac.uk/ portal/en/publications/health-care-costs-in-the-english-nhs(afae ffbd-5d6b-42f9-913d-ad781b4b1fc8)/export.html. Accessed 5 Aug 2017.

35. Seshamani M, Gray A. Ageing and health-care expenditure: the red herring argument revisited. Health Econ. 2004;13(4):303-14.

36. Seshamani M, Gray A. A longitudinal study of the effects of age and time to death on hospital costs. J Health Econ. 2004;23(2):217-35.

37. Kasteridis P, Street A, Dolman M, Gallier L, Hudson K, Martin J, et al. The importance of multimorbidity in explaining utilisation and costs across health and social care settings: evidence from South Somerset's Symphony Project. 2014. https://www.york.ac. uk/media/che/documents/papers/researchpapers/CHERP96_multi morbidity_utilisation_costs_health_social care.pdf. Accessed 17 Nov 2017.

38. Howdon D, Rice N. Health care expenditures, age, proximity to death and morbidity: implications for an ageing population. J Health Econ. 2018;57:60-74.

39. Briggs AH. A Bayesian approach to stochastic cost-effectiveness analysis. Health Econ. 1999;8(3):257-61.

40. Claxton K. The irrelevance of inference: a decision-making approach to the stochastic evaluation of health care technologies. J Health Econ. 1999;18(3):341-64. 
41. Rappange D, van Baal P, van Exel N. Unrelated medical costs in Life-Years gained. Pharmacoeconomics. 2008;26(10):815-30.

42. Walker S, Asaria M, Manca A, Palmer S, Gale CP, Shah AD, et al. Long-term healthcare use and costs in patients with stable coronary artery disease: a population-based cohort using linked health records (CALIBER). Eur Heart J Qual Care Clin Outcomes. 2016;2(2):125-40.

43. Curtis L. Unit costs of health and social care 2012. PSSRU; 2012. http://www.pssru.ac.uk/project-pages/unit-costs/unit-costs-2012/. Accessed 17 Nov 2017.
44. NHS Information Centre. Prescription cost analysis-England, 2012. NHS; 2013. https://digital.nhs.uk/catalogue/PUB10610. Accessed 17 Nov 2017.

45. Department of Health. NHS reference costs: financial year 2011 to 2012. 2012. https://www.gov.uk/government/publications/nhsreference-costs-financial-year-2011-to-2012. Accessed 17 Nov 2017.

46. van Baal PHM, Hoogendoorn M, Fischer A. Preventing dementia by promoting physical activity and the long-term impact on health and social care expenditures. Prev Med. 2016;85:78-83. 